\title{
Microcontact Processing for Microtechnology and Biology
}

\author{
Emmanuel Delamarche* \\ Werner Prize Winner 2006
}

\begin{abstract}
Processes occurring on surfaces are ubiquitous both in everyday life and in the research activities of many scientists. When these processes can be mastered with sufficient spatial resolution, they are employed for the manufacture of electronic devices, displays, data storage equipment, optical equipment, sensors, diagnostic devices, etc. A new class of surface-patterning techniques in which chemistry is localized by means of micropatterned stamps or microfluidic elements is emerging. These techniques might be increasingly applied to complement 'conventional' microfabrication techniques and to create high-performance, miniaturized bio-analytical systems.
\end{abstract}

Keywords: Immunoassays · Microcontact printing · Microfluidics · Self-assembled monolayers · Soft lithography

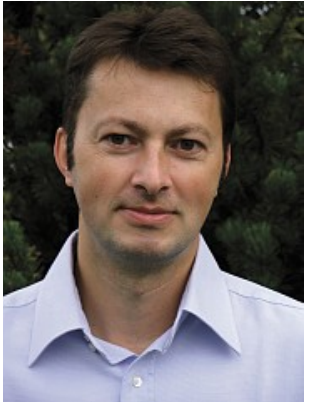

Emmanuel Delamarche, born 1966 in Nancy, France, studied chemistry at the University Paul Sabatier of Toulouse, where he received a degree in supramolecular chemistry in 1992 . He then moved to Switzerland to make his $\mathrm{PhD}$ in biochemistry at the IBM Zurich Research Laboratory under the supervision of Dr. B. Michel (IBM), Dr. H. Sigrist (Univ. Bern) and Prof. H. R. Bosshard (Univ. Zurich). He received his $\mathrm{PhD}$ in 1995 for work done on the (photo)attachment of biomolecules on self-assembled monolayers. He was post-doctorate until 1997 when he became a research staff member. He is now leading work on experimental biosciences

\footnotetext{
${ }^{\star}$ Correspondence: Dr. E. Delamarche

Zurich Research Laboratory

Säumerstrasse 4

$\mathrm{CH}-8803$ Rüschlikon

Tel.: +4144724 8283

E-Mail: emd@zurich.ibm.com
}

at the IBM Zurich Research Laboratory. His research focuses on developing novel surface-patterning techniques based on soft lithography and self-assembly for microfabrication processes and for achieving high-performance bio-analytical systems. His current projects deal with the fast and sensitive detection of multiple markers for heart diseases by means of portable microfluidics and the efficient development of vaccines against H5N1-type viruses in a joint project with the Scripps Research Institute. In addition, Dr. Delamarche acts as a research expert for IBM in internal task forces and studies as well as in consulting activities. He has received several awards from IBM and was named 'Master Inventor' by the IBM Research Division in 2002.

\section{Introduction}

Structuring and patterning surfaces are central to many applications, including the fabrication of microelectronic devices (microprocessors, memory chips, charge-coupled devices, printed circuit boards, etc.), displays, micro electrochemical systems, sensors, lab-on-a-chip devices, and other bioanalytical devices. ${ }^{[1]}$ The workhorse for structuring surfaces is photolithography, in which a photoresist that is spun-coated on a substrate is exposed in some areas to ultraviolet light using an optical mask (Fig. 1). Exposure to light changes the physicochemical properties of the photoresist, which can then be selectively removed during a development step. The photoresist left on the substrate acts as a physical mask to protect the substrate from etchants. Photolithography has been developed to an exquisite level of precision during the past decade. It can now be used for patterning features as small as $90 \mathrm{~nm}$ for production and $65 \mathrm{~nm}$ for research and development, on $\mathrm{Si}$ wafers $300 \mathrm{~mm}$ in diameter, and with impressive accuracy, process control, and throughput. ${ }^{[2]}$ However, this technique is expensive as it requires costly instruments, ultrapure reagents, and an environment free of particulates.

The invention of microcontact printing $(\mu \mathrm{CP})$ by Whitesides and colleagues in 1993 provides an exciting complementary technique to photolithography for the chemical functionalization and structuring of surfaces with spatial control (Fig. 1). ${ }^{3]}$ The central element in $\mu \mathrm{CP}$ is an elastomeric stamp, which is molded using a lithographically-prepared mold. Inking the stamp with a solution of monolayer-forming molecules allows the spontaneous formation of a selfassembled monolayer (SAM) in the regions of contact between the stamp and substrate. Microcontact printing departs from conventional lithography for several reasons. The elastomeric nature of the stamp minimizes the lateral extension of defects due to particles contaminating stamps, inks and substrates. Large and/or curved substrates can be microcontact-printed. In addition, very different types of active ink components can be used, including alkanethiols, silanes, inorganic complexes, dendrimers, colloids, beads, proteins, polymers, and even metallic layers. These inks can be used to protect a substrate from wet etching, to change the 


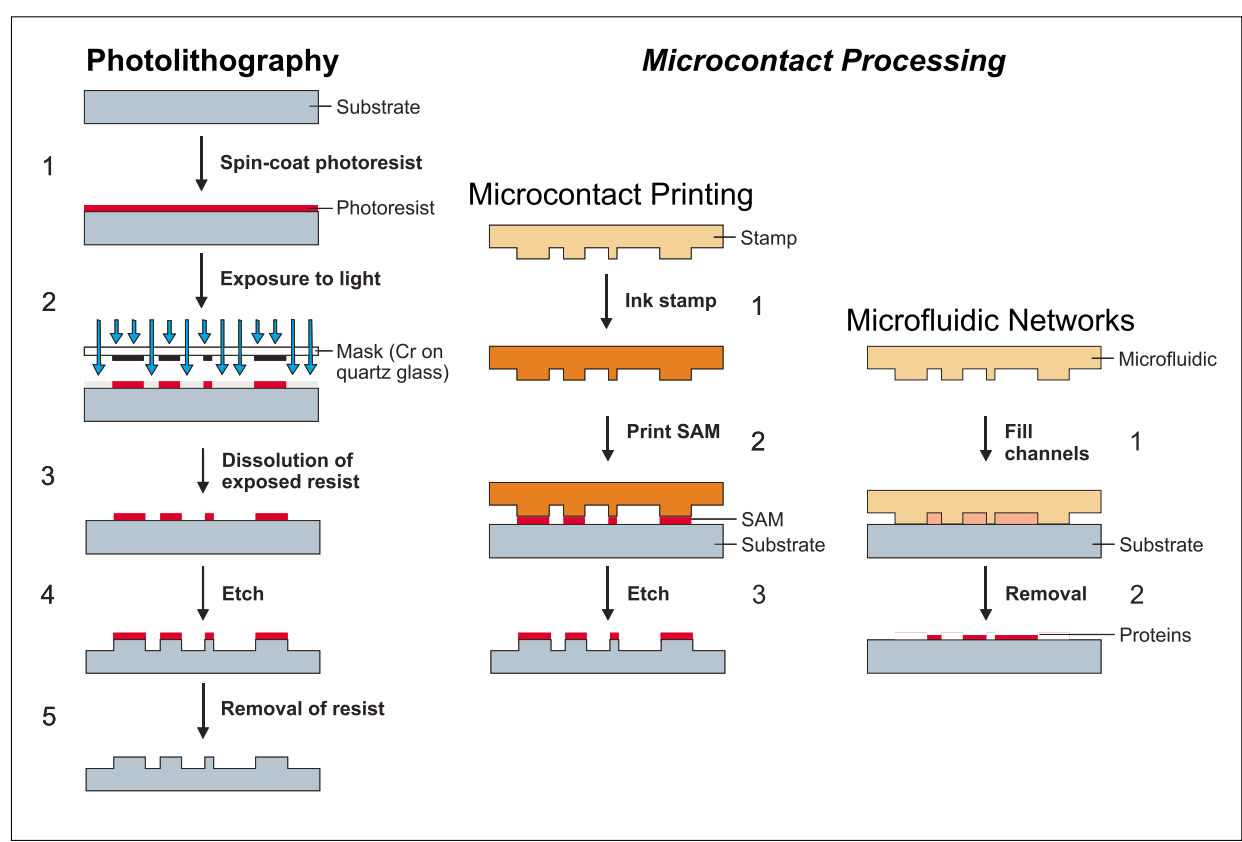

Fig. 1. Microcontact processing refers to concepts derived from microcontact printing, in which a patterned elastomeric stamp is used to microcontact print an ink on a surface instead of using optical masks, photoresists and ultraviolet light as conventionally done in photolithography. With microfluidic networks, the adhesion of a stamp to a surface ensures efficient reversible sealing, which allows one to expose areas of the surface to chemicals and biological solutions.

wetting properties of surfaces, to catalyze the electroless deposition of metal ions from solution, or to detect analytes in solutions. ${ }^{[4]}$

Microcontact printing has evolved in a number of variants, collectively termed 'soft lithography', ${ }^{[5]}$ which together provide convenient means for patterning surfaces for research applications and potentially for shifting the fabrication paradigms of, for example, flat panel displays, diffractive optical elements, and organic-based electronic devices. Microcontact printing is clearly versatile and can effect additive and subtractive patterning processes, and pattern substrates with sub-micrometer resolution with relative ease. Alternatively to printing, patterned elastomers can reversibly seal areas of a surface for local processing of the areas. ${ }^{[6,7]}$ One such variant is termed 'microfluidic networks' and can pattern biomolecules on surfaces (Fig. 1). ${ }^{[7]}$

In this article, $\mu \mathrm{CP}$ and microfluidic networks are referred to as microcontact processing. This may help emphasize that the processing steps that follow contacting a surface with a patterned elastomer also play a crucial role for achieving high-quality patterns. Two examples are reviewed below showing that microcontact processing can localize chemical processes on surfaces that otherwise are challenging if conventional lithography techniques are used. Each example has two facets: a conceptual facet and a more application-oriented facet. The first example deals with the selective etching of metals such as $\mathrm{Cu}$ using microcontact-printed SAMs and the patterning of $\mathrm{Cu}$ electroless deposited on large glass substrates. The second example shows how to pattern proteins on surfaces accurately using microfluidic networks for the miniaturization of surface immunoassays.

\section{Limits of Self-assembled Monolayer Resists}

Poly(dimethylsiloxane) (PDMS) is a material of choice for stamps. This elastomer forms good spontaneous contact with many surfaces, is commercially available (e.g. Sylgard $184^{\mathrm{TM}}$ ), is transparent as well as resistant to many chemicals, and can be thermally cured on molds within a few hours when heated at $60^{\circ} \mathrm{C}$. Structures such as lines and posts can be replicated down to approximately $60 \mathrm{~nm}$ in PDMS materials that are harder than Sylgard 184. ${ }^{[8]}$

Molecules capable of forming SAMs on surfaces by adsorption from solution can also be used as ink in $\mu \mathrm{CP}$ if two criteria are met. First, the molecules should preferably be soluble in a solvent compatible with PDMS. ${ }^{[9]}$ Solvents such as heptane and tetrahydrofuran considerably swell PDMS stamps unlike water and ethanol. Ethanol is a convenient solvent because it is readily removed from stamp by drying the inked stamps using a stream of nitrogen. Second, the ink molecules should have some affinity for PDMS in order to be retained within the stamp or on its surface in sufficient amounts for at least one print. Several ink/substrates systems have been devised and studied in depth. These include alkanethiols and disul- fides on $\mathrm{Au}, \mathrm{Cu}, \mathrm{Ag}$, and $\mathrm{Pd}^{[10-12]}$ as well as trichlorosilanes and siloxanes on silicon dioxide and glass. ${ }^{[13,14]}$ Modifying the surface chemistry of PDMS using strong oxidative treatment makes it possible to ink also polar metal complexes, polymers, peptides, oligonucleotides, and dendrimers. ${ }^{[15]}$

Alkanethiols such as hexadecanethiol (HDT), octadecanethiol, or eicosanethiol (ECT) fulfill both conditions and have the best potential for practical applications of $\mu \mathrm{CP}$. HDT is soluble in ethanol, commercially available and cheap, easy to purify using chromatography and recrystallization, and has good affinity for PDMS. ${ }^{[16]}$ The main steps needed to pattern alkanethiols on noble metals are depicted in Fig. 2A. After inking and drying the stamp, alkanethiols transfer to the substrate in the regions of contact during the printing step. Printing can be done by hand or using a tool. There is no need to apply pressure to the stamp during printing: PDMS spontaneously adheres to surfaces owing to adhesion. Once the stamp has been removed from the surface, the printed SAMs can be used to control the surface properties of the substrate such as its wettability, adherence, chemical reactivity, protein repellency, or resistance to corrosion. ${ }^{[4]}$

SAMs patterned on $\mathrm{Au}, \mathrm{Cu}, \mathrm{Ag}$ and $\mathrm{Pd}$ can locally protect the underlying substrate from wet etching (Fig. 2B). Etchants for Au are cyanide, ferricyanide, or thiosulfate, for example. ${ }^{[5]} \mathrm{Cu}$ can be etched using an aqueous solution of ferrichloride. A SAM of HDT on $\mathrm{Au}$ has a thickness of $2 \mathrm{~nm}$ and is typically used to etch $\mathrm{Au}$ in a depth of 20 to $50 \mathrm{~nm}$. Despite the relatively high order and coverage of SAMs microcontact printed on $\mathrm{Au}$, the selectivity of the etch process (defined as the ratio between the thickness of the monolayer resist and the maximal practical depth of the etched structures) is relatively limited for several reasons. First, SAMs are imperfect. They have regions of lower density, such as domain boundaries, and single molecules missing in crystalline domains. ${ }^{[17]}$ Polycrystalline substrates also limit the degree of molecular order in SAMs. Second, a depletion of ink molecules may occur in some areas of the pattern during printing. ${ }^{[18]}$ Finally, molecules in SAMs can reversibly desorb during the rinsing steps performed after printing, and stray light and oxygen can oxidize thiol headgroups into sulfonates. Each missing molecule in a microcontact printed SAM might represent a path to the substrate for etchants from solution (Fig. 2C). Such defects are not averaged out by the presence of multilayers of molecules unlike in conventional resists. These problems are exacerbated when $\mathrm{Cu}$ is used as a substrate owing to the presence of a copper oxide layer, which interferes with the formation of the alkanethiol SAM during printing. 


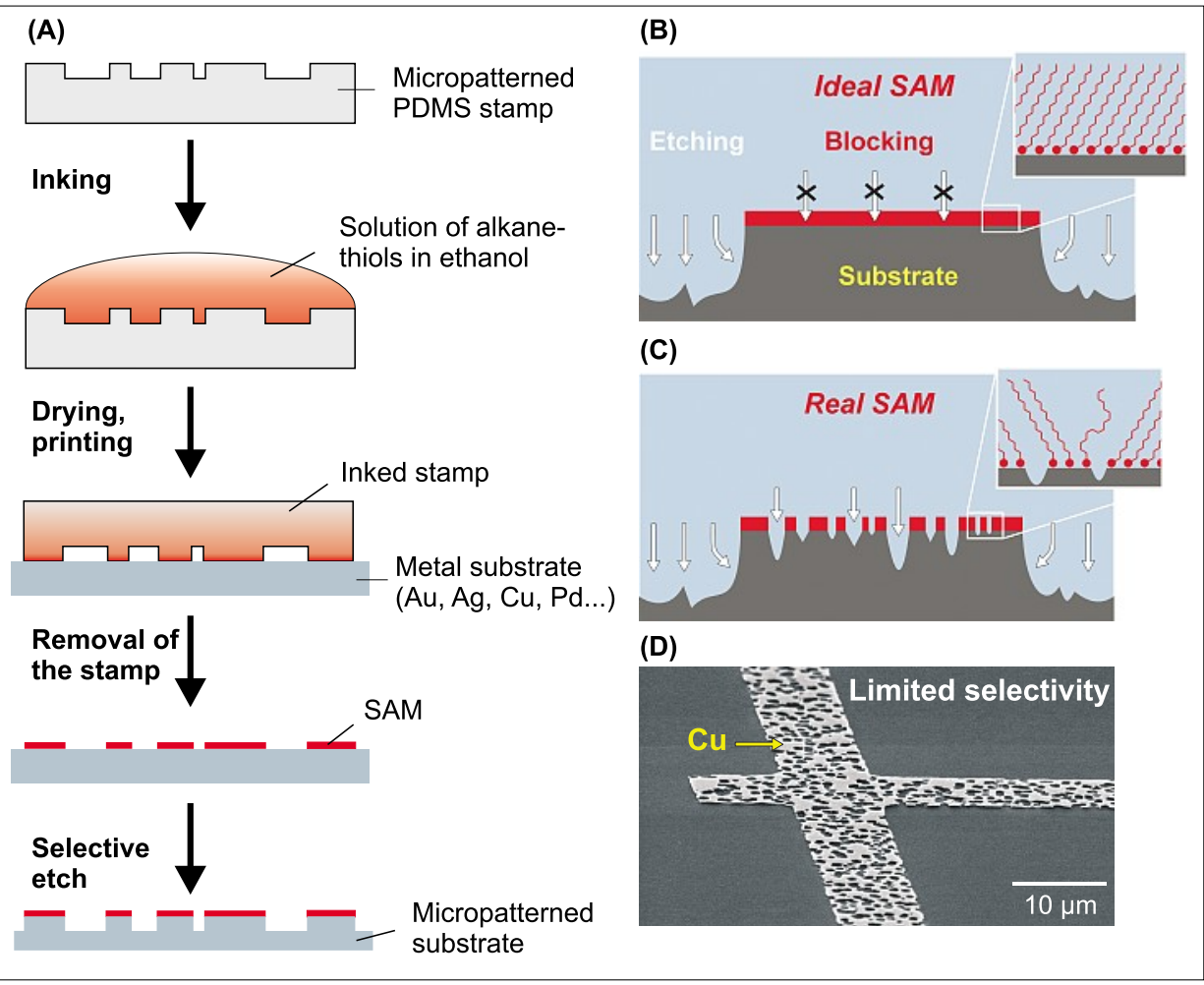

Fig. 2. Structuring substrates using $\mu \mathrm{CP}$ and wet etching $(\mathrm{A})$ relies on the efficient protection of a microcontact printed substrate by a SAM (B). Defects in SAMs, however, provide paths for etchants (C), which can result in patterns having limited quality as revealed in the scanning electron microscope image in (D).

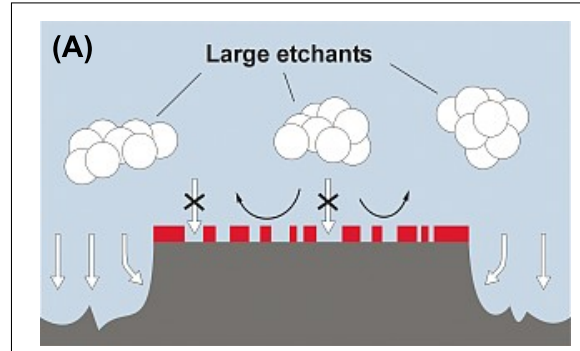

(B)
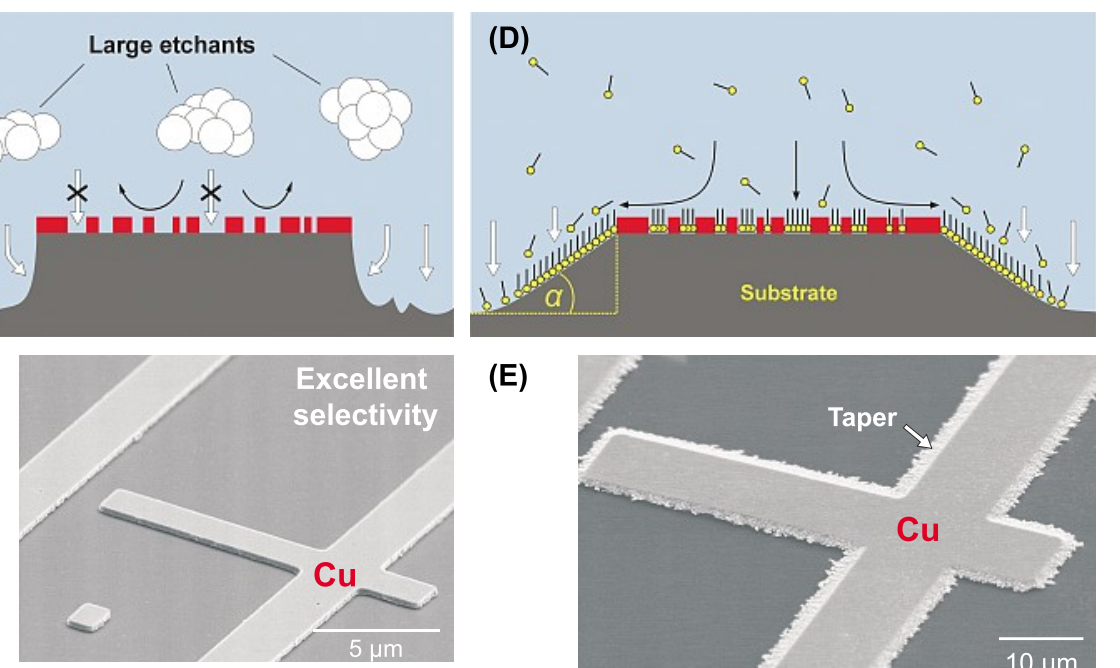

(E)

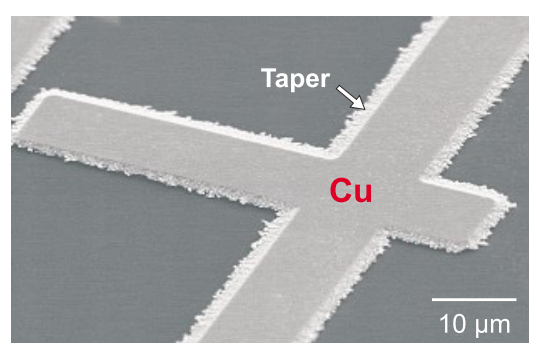

(C)

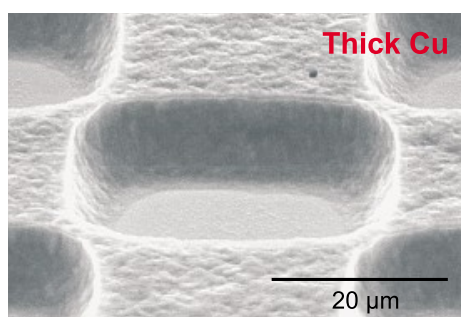

(F)

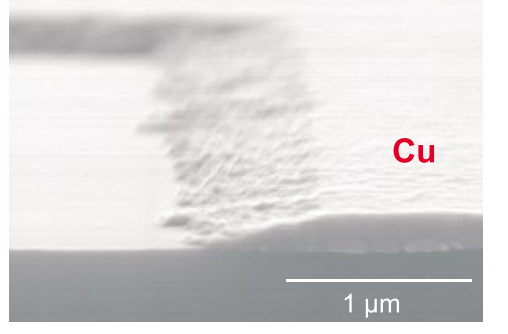

Fig. 3. A selective wet etch chemistry for $\mu \mathrm{CP}$ employs a small oxidizer (NBSA, depicted by the white arrows) and bulky carrier (PEl, large clusters), (A). The oxidized metal can only be removed from the surface following complexation by the carrier. If the carrier is sufficiently large compared with the average size of defects in the SAM, a selective etch process is ensured, as evidenced by the highquality patterns formed in 1- $\mu \mathrm{m}$-thick (B) and 2.2- $\mu \mathrm{m}$-thick Cu layers (C). An alternative strategy is to add molecules that have affinity for defects in SAMs to an etch bath (D). If these molecules are lipophilic such as HDT, they can diffuse sideways from the regions of contact and compete with etching so as to form tapered sidewalls $(\mathrm{E})$ and $(\mathrm{F})$.
This is evidenced in Fig. 2D, where a 200nm-thick $\mathrm{Cu}$ layer on a Si wafer was microcontact printed with ECT and immersed in a $0.1 \mathrm{M} \mathrm{CN}^{-} / \mathrm{O}_{2}$ etch bath.

Patterning alkanethiols on surfaces with sub-micrometer accuracy requires short printing times and a limited ink concentration to minimize the surface diffusion of alkanethiols away from the regions of contact. ${ }^{[19]}$ Therefore, the completion of SAMs during short printing steps might not be achievable for some pattern geometries, and in these cases selective etch chemistries are particularly important. ${ }^{[20]}$

\subsection{Hyper-selective and Directional Wet-etching}

$\mathrm{Cu}$ is of particular importance in the microelectronic industry because it is one of the best conductors at room temperature, it is cheap, ductile, and can be deposited using evaporation, sputtering, electrochemical processes or electroless deposition. $\mathrm{Cu}$ on surfaces cannot be patterned using reactive ion etching, but must be patterned using wet etch processes. A selective wet etch chemistry for $\mathrm{Cu}$ microcontact printed with SAMs that is based on self-assembly processes occurring during wet etching is shown in Fig. 3A. [21]

In a first example, $\mathrm{Cu}$ is oxidized by a small molecule, 3-nitro benzene sulfonic acid (NBSA), which does not yet lead to well soluble $\mathrm{Cu}^{2+}$ or $\mathrm{Cu}^{+}$complexes. A branched polyethylenimine (PEI) polymer having a molecular weight of $\sim 800 \mathrm{~g} \mathrm{~mol}^{-1}$ then complexes the oxidized $\mathrm{Cu}$ via its primary and secondary amino groups, which results in well soluble $\mathrm{Cu}$ complexes that are able to diffuse away from the surface. Bulky and polar PEI molecules are unlikely to penetrate through defects in SAMs. Using this strategy, a 2.5-nm-thick monolayer of ECT can protect up to $2.2-\mu$ m-thick $\mathrm{Cu}$ layers (Fig. 3B and C). This represents an etch selectivity of 1:800, which is not encountered when organic resists are used - not even with costly and complex dry etch systems.

Patterning surfaces with control of the edge profile is important for stacking numerous layers with good adhesion and homogeneity. Etching single crystals along preferential directions is well known but is challenging for polycrystalline materials such as evaporated metals. A strategy for tapering $\mathrm{Cu}$ using an advanced wet etch chemistry is shown in Fig. 3D. In this second example, an alkaline bath containing $\mathrm{CN}^{-}$and dissolved oxygen is used to etch $\mathrm{Cu}$ in the regions where no SAM of ECT was printed. High selectivity is achieved by adding HDT to the bath at micromole concentration. HDT inserts itself into defects in the ECT monolayer and 'heals' defects. The hydrophobic regions where ECT is printed also act as 'antennas' upon which 
HDT from the bath accumulates and diffuses sideways. Accordingly, a competition between a laterally spreading monolayer of HDT and etching of $\mathrm{Cu}$ ensues. This competition results in a taper of the sidewalls with an angle that primarily depends on the concentration of HDT additives and the strength of the etch bath $\left(\mathrm{CN}^{-}\right.$concentration) (Fig. 3E and F). ${ }^{[21]}$ Here again, conventional etch systems are in principle not able to control the edge profile of polycrystalline structures with such a high selectivity and precision.

\subsection{Patterning Cu for Flat Panel Display Applications}

Microcontact processing can be used for additive patterning where, instead of etching a material, a metal or alloy is deposited from solution onto a surface with spatial control.[22] Electroless deposition (ELD) is a widely used technique for the deposition of metals from solution onto non-conductive substrates. ${ }^{[23]}$ ELD relies on the spontaneous reduction of metal ions by a reducing agent in solution. ELD baths are thermodynamically unstable but kinetically stabilized by adding strong ligands for the metal ions. A catalyst on the insulating surface is needed to start the deposition process, which then proceeds autocatalytically. Typical metals in electroless deposition are $\mathrm{NiB}, \mathrm{NiP}, \mathrm{NiWP}, \mathrm{Cu}, \mathrm{CoP}$ and, to a lesser extent, $\mathrm{Au}$ and Ag. Pd-rich particles such as $\mathrm{Pd} / \mathrm{Sn}$ colloids are commonly used as a catalyst for ELD. ${ }^{[23]}$ The electroless deposition of $\mathrm{Cu}$ onto a glass substrate and its subsequent patterning using $\mu \mathrm{CP}$ could be of interest for the fabrication of gate patterns for thin film transistor (TFTs) arrays for liquid crystal displays (LCDs). TFTs in LCDs orient liquid crystals to attenuate or block the transmission of light from the back of the display to the outside. Each TFT in an array is addressed by driving current in one vertical and one horizontal conductive line. The lines in contact with the glass substrates are usually the gate lines, which address the gates of the TFTs. ${ }^{[24]} \mathrm{Cu}$ is a good candidate metal for gate patterns: well conductive gate lines can be made narrower so that more light passes through the TFT array while enabling fast switching of the TFTs. Large metal-sputtering and lithographic equipment is used for the deposition and patterning of the TFT metals. Exchanging the vacuum deposition of $\mathrm{Cu}$ with electroless deposition and the photolithography steps for patterning $\mathrm{Cu}$ with $\mu \mathrm{CP}$ may

i) lead to cheaper processes,

ii) help when using larger glass substrates, iii) reduce the consumption of chemicals,

iv) reduce the footprint of equipment in a factory, and

v) increase patterning throughput.

A proof of principle for the ELD of $\mathrm{Cu}$ and patterning using $\mu \mathrm{CP}$ is shown in Fig.
4A. ${ }^{25]}$ From the beginning, this process was developed with the goals of not using inflammable solvents, of not using cyanidecontaining baths for etching microcontact printed $\mathrm{Cu}$, and of having a large process window. Display glass substrates of $15 \times$ 15 sq. inch in size were used. First, a glass substrate is functionalized with 3-(2-amino-ethylamino)-propyl-trimethoxysilane (EDA-Si) by immersing the glass in a $1 \%$ solution of EDA-Si in water for $10 \mathrm{~min}$, rinsing it with water and drying it. EDA-Si hydrolyzes within a few seconds in water but remains able to bind glass through electrostatic interactions between its charged amino groups and deprotonated silanols $\left(-\mathrm{Si}_{-} \mathrm{O}^{-}\right)$from the glass surface. During drying, condensation reactions occur between hydrolyzed EDA-Si molecules and silanols from the glass surface, resulting in a $\sim 0.5$ nm-thick monolayer on the glass surface. ${ }^{[26]}$ Next, the derivatized glass is immersed for $30 \mathrm{~s}$ in an acidic solution containing $\mathrm{Pd} / \mathrm{Sn}$ particles, which results in the homogeneous deposition of ELD catalyst on the glass surface. The glass is then briefly immersed in an aqueous bath of $\mathrm{HBF}_{4}$ to remove some

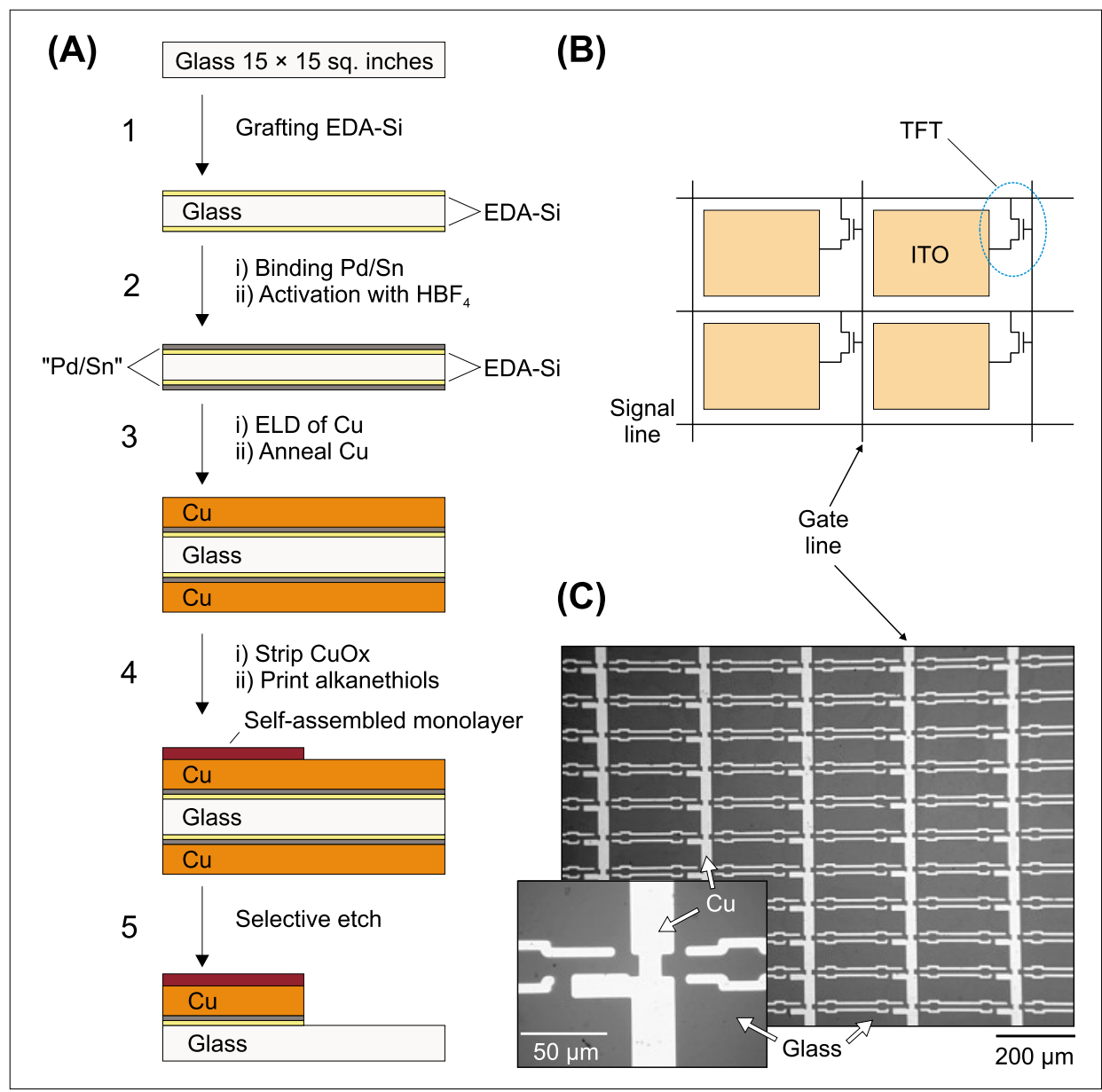

Fig. 4. Combining ELD and $\mu \mathrm{CP}$ for patterning $\mathrm{Cu}$ on large substrates is a plausible alternative to the patterning of vacuum-deposited Cu films using photolithography. (A) This patterning method is based on highly controlled, spontaneous chemical processes such as the functionalization of glass with EDA-Si and Pd/Sn catalysts, ELD of $\mathrm{Cu}$, forming a SAM of HDT using $\mu \mathrm{CP}$, and selective etching. (B) Gate structures for TFT arrays can be patterned with high accuracy, as shown in the micrograph in (C). of the Sn and better expose the Pd catalytic core to reactants in the ELD bath. Deposition of $\mathrm{Cu}$ proceeds in a commercial ELD bath (Cu Thru CUP bath from Yuemura), the $\mathrm{pH}$ of which was lowered to 12.0 to minimize hydrolysis of the siloxane bonds between EDA-Si and glass by hydroxides. Typically, $120 \mathrm{~nm}$ of $\mathrm{Cu}$ was deposited on both faces of the glass, and HDT was microcontact printed using a large micropatterned stamp as well as a specially designed printing tool. ${ }^{[25]}$ Undesired $\mathrm{Cu}$ on the back of the glass and in the non-printed regions was removed by means of a selective etch chemistry using NBSA and PEI. The optical microscope image in Fig. $4 \mathrm{C}$ shows a part of a pattern comprising $1024 \times 768$ pixels, which corresponds to a 12.1-in. XGA type of display.

The first part of this paper showed how to revisit some well-established surface patterning processes using microcontact

\section{Miniaturizing Biological Assays Using Surface Processing}


processing and self-assembling systems. This second part describes how microcontact processing enables patterning proteins on surfaces for the miniaturization of biological assays. ${ }^{[27]}$ Most of the proteins must have a well-defined, three-dimensional native structure to fulfill their function properly. Proteins on surfaces play an important role in bioanalysis, cellular adhesion and tissue engineering, for example. However, proteins are challenging to pattern on surfaces because they are readily denatured by organic solvents, ultraviolet light and heat. The conformal contact that occurs when PDMS is placed on another surface provides the opportunity to seal microfluidic elements with PDMS (Fig. 5A). The microfluidic elements can be soft (PDMS replica) ${ }^{[7]}$ or hard (microfabricated in $\mathrm{Si}$ or glass). ${ }^{[28]}$ The latter possibility may be more interesting because it allows a range of wettable microstructured materials to be filled using capillary forces: PDMS microfluidics must be made hydrophilic unless they are filled by means of active pumping principles. As solutions of protein are displaced in microchannels, proteins spontaneously adsorb from solution to form lines on a hydrophobic PDMS substrate. This deposition method can be used to pattern lines of capture antibodies for miniaturized immunoassay, in which antigens captured from solution are detected using a second, fluorescently-labelled antibody (Fig. 5B).

The laminar flow of solutions in microfluidics helps keep reactants well separated from aliquots owing to the absence of turbulent mixing so that they can be successively drawn into a microfluidic system. Diffusion of reactants in microfluidics is passive (Fig. 5C) unless specifically designed mixers are placed in the microfluidic flow paths. The accurate placement of protein receptors on surfaces, such as antigens, antibodies, enzymes, cell adhesion molecules, is key for high-performance assays when these receptors are used for detecting analytes from samples. An accurate pattern of receptors translates into a clear pattern of surface-bound analytes and therefore of well-defined fluorescent signals. Miniaturized receptor areas are also helpful for detecting various analytes in parallel using minimal volumes of samples and reagents. Fig. 5D shows the high-resolution capability of microfluidics for patterning proteins on surfaces. In this image, a solution of 200 $\mu \mathrm{g} \mathrm{ml}^{-1}$ of fluorescently-labelled antibodies in PBS was drawn into 5- $\mu \mathrm{m}$-wide $\mathrm{Si}$ microchannels using capillary forces. Lines of densely deposited antibodies formed in only $5 \mathrm{~min}$ and with an edge resolution of $\sim 200 \mathrm{~nm} .{ }^{[29]}$ Microfluidics harbor the risk of depletion of reactants, however. Fig. 5E shows surface density gradients of antibodies on PDMS, which resulted from depleting the antibody solution confined in the mi-

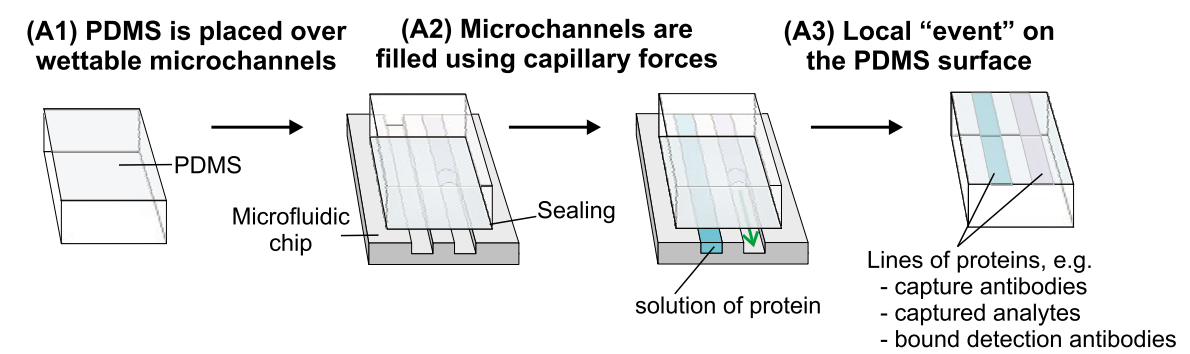

(B) Fluorescence surface immunoassay

(D) Proteins patterned using a microfluidic network and a stamp
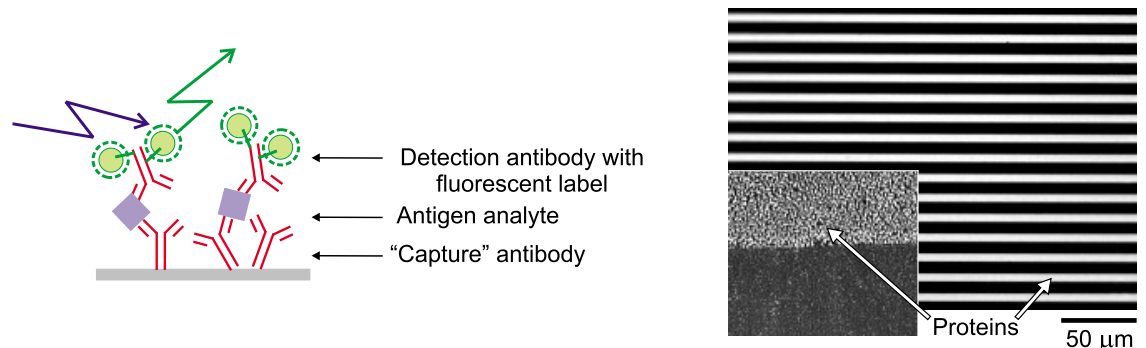

(C) Laminar flow in microchannels

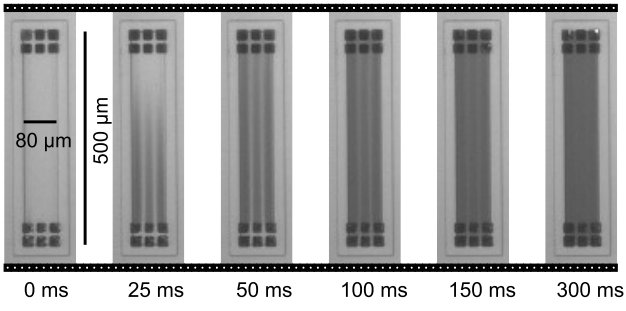

(E) Surface-density gradient of proteins on glass

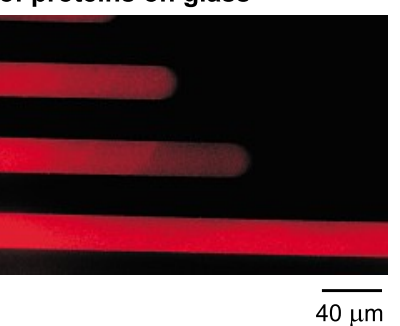

Fig. 5. Microcontact processing of surfaces with solutions of proteins takes advantage of the efficient sealing that occurs when PDMS contacts microfluidic elements. Patterning PDMS with lines of biomolecular receptors $(A)$ permits fluorescence immunoassays to be miniaturized (B). (C) At the micrometer scale, the flow of processing solutions and rinsing liquids is laminar, and thus passive diffusion limits the spreading of reactants between parallel flow streams. (D) Fluorescence microscope and atomic force microscope (inset) images illustrating that microfluidics can pattern proteins with very high resolution on PDMS, from where they can then be printed onto a glass substrate. (E) The high surface-to-volume ratio of microfluidic channels may result in the depletion of reactants as evidenced by the surface density gradient of fluorescently-labelled antibodies deposited on PDMS (E).

crochannels during the deposition process. Surface density gradients may help study adhesion or motility processes of living cells. In biological assays, depletion effects must be prevented to ensure that the signals measured on surfaces accurately reflect the concentrations of analytes in samples.

\subsection{Capillary-driven Microfluidics}

The use of capillary forces in microfluidics to pattern proteins on surfaces or to detect analytes from samples was refined by encoding advanced functions into microfluidic chips. These chips are called autonomous capillary systems (ACSs) to emphasize that they do not need external actuators or peripheral equipment to operate (Fig. 6A). ${ }^{[28,30]}$ The most important functional element of these chips are the loading pads, into which solutions are pipetted, the connecting microchannels, the reaction chambers, where the assays are localized, the capillary retention valves to prevent trapping air or adventitious drying in the reaction chambers, the vias through the silicon wafer, and the capillary pumps having arrays of hydrophilic microstructures for efficiently pumping all liquids added to the loading pads. By coating the chips with a thin layer of Au, hydrophobic SAMs of HDT can be printed around the microstructures, and poly(ethylene glycol)functionalized alkanethiols can be deposited inside the microstructures to render the latter hydrophilic and protein-repellent.

The immunoassay shown in Fig. 6B for the detection of the general marker for inflammation or infection, the C-reactive protein (CRP), has several interesting features. First it was completed in less than 25 min because the steps, which are not kinetically-limited (e.g. rinsing), take only a few seconds. No depletion of reactant occurred because sufficient reactant was flushed 


\section{(A) Autonomous capillary system}

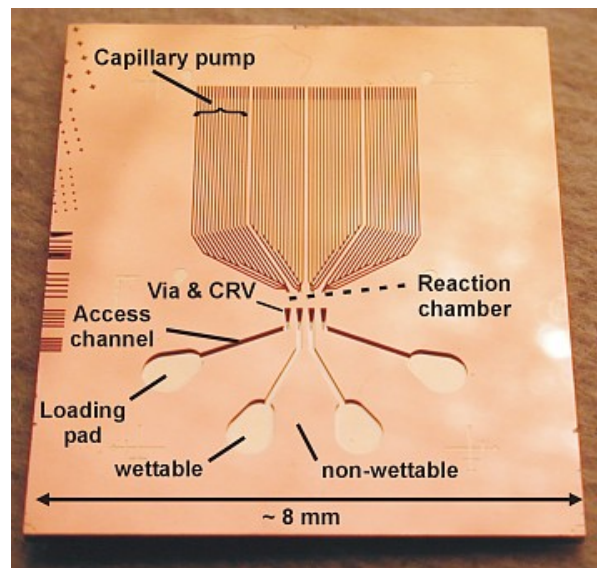

\section{(B) Surface fluorescence immunoassay}

1

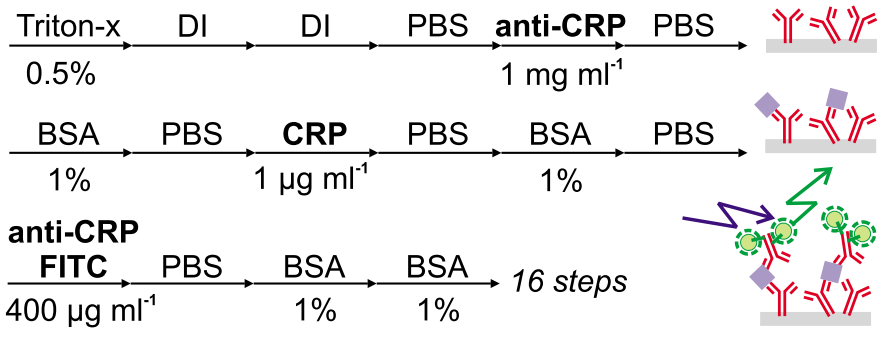

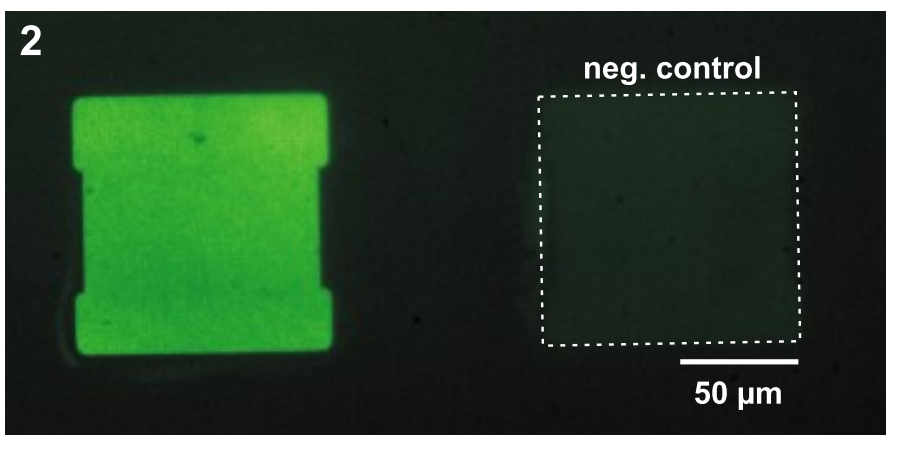

Fig. 6. ACSs for high-performance surface immunoassays. (A) Image of an ACS microfabricated in Si and coated with Au that has independent flow paths starting with pads in which aliquots are initially pipetted and ending with capillary pumps. Immunoassays are done on the other side of the chip in four reactions chambers that are sealed by a slab of PDMS (not visible in this image). (B) The aliquots of reagents, rinsing liquids, and samples are successively displaced through the reaction chamber to effect sandwich surface fluorescence immunoassays. The results appear as fluorescence signals on the surface of PDMS.

through the reaction chamber. The volume of the aliquots was $\sim 200 \mathrm{nl}$. The fluorescence signal on the PDMS surface has a clear footprint and relates to the concentration of CRP in the sample that passed in the reaction chamber. Several assays can be done in parallel, including negative and positive controls.

\subsection{Micromosaic Immunoassays}

The detection of analytes at low concentration and from small sample volumes is challenging because it requires a sensitive bioanalytical system that can accurately handle volumes of liquids in the microliter range. Microfluidic chips having planar and parallel ACSs provide a solution to this challenge if they are used in pairs (Fig. 7). One chip can be used to pattern lines of different types of capture antibodies on PDMS, for example. A series of antigens in samples can be screened using a second chip by flowing the samples across the lines of capture antibodies. This miniaturized, combinatorial assay was termed micromosaic immunoassays because a mosaic of fluorescent signals on the PDMS surface at the end of the assay reveals which antigen was present in each sample and bound to the corresponding capture antibody. ${ }^{[31]}$ Evaporation of liquid from the loading pad is prevented or induced in the capillary pumps by cooling the pads to temperatures just above the dew point or heating the pumps, respectively. Fig. 7C shows a mosaic of fluorescence signals corresponding to an assay in which a capture antibody against the biologically important cytokine TNF- $\alpha$ was homogeneously deposited on PDMS. A solution containing a known (standards) or unknown (samples) concentration of TNF- $\alpha$ was delivered using a first chip (vertical direction), and fluorescently-labelled detection antibodies were delivered using a second chip (horizontal direction). In this example, rather than screening for more than one analyte, the effect of the concentration of the detection antibody on the sensitivity of the assay was investigated. This assay has a sensitivity reaching $\sim 20$ pg ml-1, and only necessitated $\sim 600 \mathrm{nl}$ of sample and $45 \mathrm{~min}$ for all pipetting, rinsing and incubation steps. ${ }^{[32]}$

\section{Concluding Remarks}

Non-conventional surface processing techniques have an increasing role to play in microtechnology by, for example, controlling the properties of surfaces via self-assembly and microcontact printing. These properties can be the wettability of a surface, its resistance to corrosion, its catalytic activity for electroless deposition, or its optical and electrical properties, for example. These emerging techniques might also be more amenable than conventional ones for the processing of substrates having unusual characteristics (large in area, ultrathin, fragile, hybrid in composition, flexible, or topologically complex). Novel processes for functionalizing surfaces can also help research in life sciences and routine diagnostic applications by miniaturizing biological assays. In this case, the positioning on surfaces of biomolecular receptors can be done using microfluidics, microcontact printing, or variants of soft lithography in general. Although it is clear that miniaturization brings benefit to bio-analytical systems by reducing sample and reagents consumption, improving the portability of analytical systems, and generating results in less time and sometimes with higher quality than non-miniaturized systems, a great challenge is to develop high-performance bio-analytical systems that can remain simple enough for use by non-experts and that can build on existing practices and technological platforms. I think that chemists are well poised to help solve these challenges and realize these op- 
(A) Principle of a micromosaic immunoassay

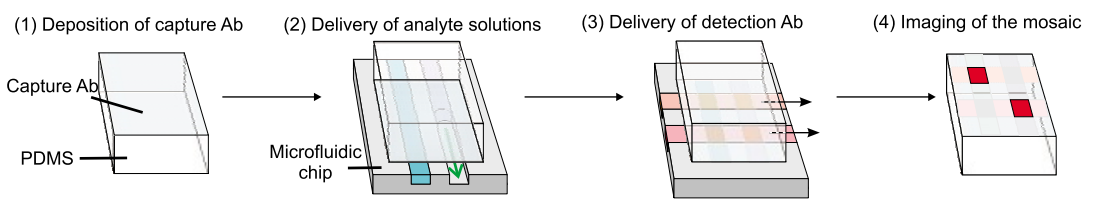

(B) Microfluidic chip with 11 capillary systems

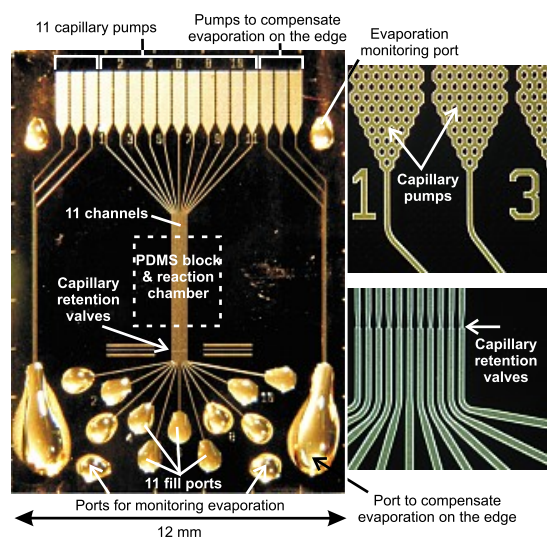

(C) Detection of TNF- $\alpha$ with 1 pM sensitivity

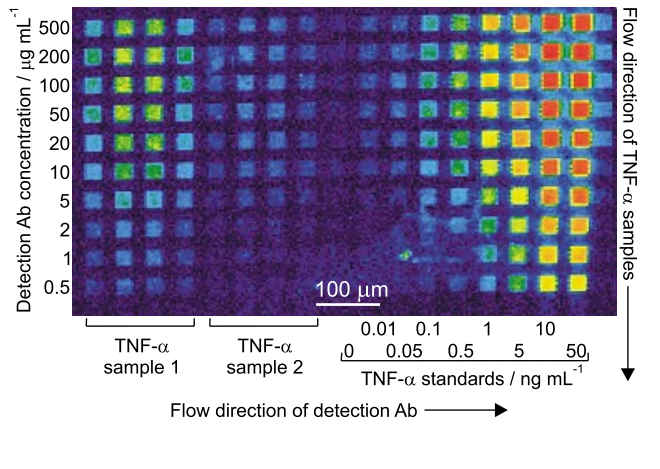

Fig. 7. Micromosaic immunoassays. (A) These combinatorial assays require two chips for screening a series of ligands in solution using lines of receptors on a surface. (B) Example of a Si chip coated with Au and having eleven independent ACSs. Each loading pad has $\sim 300 \mathrm{nl}$ of solution. The PDMS substrate (not shown here) for an assay would be placed in the central region, where the microchannels of each ACS run parallel. (C) Fluorescence scanner image showing where TNF- $\alpha$ was captured and detected on the PDMS surface.

portunities, in particular by working with a multidisciplinary approach.

\section{Acknowledgments}

I would like to express my deepest gratitude to B. Michel and H. Biebuyck, who started the microcontact processing project at IBM, for their guidance and advice. Teamwork has been invaluable in achieving the results described above, and I am particularly indebted to A. Bernard, M. Geissler, D. Juncker, H. Schmid and H. Wolf, as well as to many other colleagues. I gratefully acknowledge continuous support from P. F. Seidler and W. Riess, and financial support from IBM, the Swiss National Science Foundation, the Swiss Commission for Innovation and Technology, and the Swiss Federal Office of Education and Science in the framework of EC-funded projects (4th, 5th, and 6th European Framework Projects).

\section{Received: December 18, 2006}

[1] M. J. Madou, Fundamentals of Microfabrication, CRC Press, New York, 2002.

[2] The International Technology Roadmap for Semiconductors: 2006 Update, available at http://www.itrs.net/Links/ 2006Update/2006UpdateFinal.htm

[3] A. Kumar, G. M. Whitesides, Appl. Phys. Lett. 1993, 63, 2002-2004.

[4] A. Kumar, H. A. Biebuyck, G. M. Whitesides, Langmuir 1994, 10, 1498-1511.

[5] Y. Xia, G. M. Whitesides, Angew. Chem. Int. Ed. 1998, 37, 551-575.

[6] E. Kim, Y. Xia, G. M. Whitesides, Nature 1995, 376, 581-584.
[7] E. Delamarche, A. Bernard, H. Schmid, B. Michel, H. A. Biebuyck, Science 1997, 276, 779-781.

[8] H. Schmid, B. Michel, Macromolecules 2000, 33, 3042-3049.

[9] J. N. Lee, C. Park, G. M. Whitesides, Anal. Chem. 2003, 75, 6544-6554.

[10] R. G. Nuzzo, D. L. Allara, J. Am. Chem Soc. 1983, 105, 4481-4483.

[11] P. E. Laibinis, G. M. Whitesides, D. L. Allara, Y.-T. Tao, A. N. Parikh, R. G. Nuzzo, J. Am. Chem. Soc. 1991, 113, 7152 7167.

[12] A. Carvalho, M. Geissler, H. Schmid, B. Michel, E. Delamarche, Langmuir 2002, 18, 2406-2412.

[13] J. Sagiv, J. Am. Chem. Soc. 1980, 102, 92-98.

[14] S. R. Wasserman, Y.-T. Tao, G. M. Whitesides, Langmuir 1989, 5, 1074-1087.

[15] C. Donzel, M. Geissler, A. Bernard, H. Wolf, B. Michel, J. Hilborn, E. Delamarche, Adv. Mater. 2001, 13, 1164-1167.

[16] T. E. Balmer, H. Schmid, R. Stutz, E. Delamarche, B. Michel, N. D. Spencer, H. Wolf, Langmuir 2005, 21, 622-632.

[17] E. Delamarche, B. Michel, H. A. Biebuyck, C. Gerber, Adv. Mater. 1996, 8, 719-729.

[18] T. Kraus, R. Stutz, T. E. Balmer, H. Schmid, L. Malaquin, N. D. Spencer, H. Wolf, Langmuir 2005, 21, 7796-7804.

[19] E. Delamarche, H. Schmid, A. Bietsch, N. B. Larsen, H. Rothuizen, B. Michel, H. A. Biebuyck, J. Phys. Chem. B 1998, 102, 3324-3334.
[20] J. A. Helmuth, H. Schmid, R. Stutz, A. Stemmer, H. Wolf, J. Am. Chem. Soc. 2006, 128, 9296-9297.

[21] M. Geissler, H. Schmid, A. Bietsch, B. Michel, E. Delamarche, Langmuir 2002, 18 , 2374-2377.

[22] P. C. Hidber, W. Helbig, E. Kim, G. M. Whitesides, Langmuir 1996, 12, 13751380.

[23] 'Electroless Plating: Fundamentals and Applications', Eds. G. Mallory, J. B. Hajdu, American Electroplaters and Surface Finishers Society: Orlando, FL, 1990.

[24] E. G. Colgan, P. M. Alt, R. L. Wisnieff, P. M. Fryer, E. A. Galligan, W. S. Graham, P. F. Greier, R. R. Horton, H. Ifill, L. C. Jenkins, R. A. John, R. I. Kaufman, Y. Kuo, A. P. Lanzetta, K. F. Latzko, F. R. Libsch, S. -C. A. Lien, S. E. Millman, R. W. Nywening, R. L. Polastre, C. G. Powell, R. A. Rand, J. J. Ritsko, M. B. Rothwell, J. L. Staples, K. W. Warren, J. S. Wilson, S. L. Wright, IBM J. Res. Develop. 1998, 42, 427-444.

[25] E. Delamarche, J. Vichiconti, S. A. Hall, M. Geissler, W. Graham, B. Michel, R. Nunes, Langmuir 2003, 19, 6567-6569.

[26] E. Delamarche, M. Geissler, J. Vichiconti, W. S. Graham, P. A. Andry, J. C. Flake, P. M. Fryer, R. W. Nunes, B. Michel, E. J. O'Sullivan, H. Schmid, H. Wolf, R. L. Wisnieff, Langmuir 2003, 19, 5923 5835.

[27] E. Delamarche, D. Juncker, H. Schmid, Adv. Mater. 2005, 17, 2911-2933.

[28] D. Juncker, H. Schmid, U. Drechsler, H. Wolf, M. Wolf, B. Michel, N. de Rooij, E. Delamarche, Anal. Chem. 2002, 74 6139-6144.

[29] M. Geissler, A. Bernard, A. Bietsch, H Schmid, B. Michel, E. Delamarche, J. Am. Chem. Soc. 2000, 122, 6303-6304.

[30] M. Zimmermann, H. Schmid, P. Hunziker, E. Delamarche, Lab Chip 2007, 7, 119125.

[31] A. Bernard, B. Michel, E. Delamarche, Anal. Chem. 2000, 73, 8-12.

[32] S. Cesaro-Tadic, G. Dernick, D. Juncker, G. Buurman, H. Kropshofer, B. Michel, C. Fattinger, E. Delamarche, Lab Chip 2004, $4,563-569$. 